PROCEEDINGS OF THE

AMERICAN MATHEMATICAL SOCIETY

Volume 126, Number 11, November 1998, Pages 3335-3336

S $0002-9939(98) 04715-7$

\title{
ON THE CENTER CONDITIONS OF CERTAIN CUBIC SYSTEMS
}

\author{
M. A. M. ALWASH
}

(Communicated by Hal L. Smith)

\begin{abstract}
This paper provides a new simple proof of a recent result by C. B. Collins (Differential and Integral Equations 10 (1997), 333-356) to derive the center conditions for a class of planar cubic systems. The idea is to consider periodic solutions of a related scalar non-autonomous equation.
\end{abstract}

Consider the planar system

$$
\begin{gathered}
\dot{x}=-y+x\left(\alpha x+\beta y+A x^{2}+B x y+C y^{2}\right), \\
\dot{y}=x+y\left(\alpha x+\beta y+A x^{2}+B x y+C y^{2}\right),
\end{gathered}
$$

where $\alpha, \beta, A, B$, and $C$ are real constants. In this paper, we give a simple and short proof to the following recent result of [2].

Theorem. The origin is a center for (1) if and only if

$$
A+C=0 \text { and } A \alpha^{2}+B \alpha \beta+C \beta^{2}=0 .
$$

Proof. The system (1) in polar coordinates $r$ and $\theta$ becomes

$$
\begin{gathered}
\dot{r}=p(\theta) r^{2}+q(\theta) r^{3}, \\
\dot{\theta}=1,
\end{gathered}
$$

with $p(\theta)=\alpha \cos \theta+\beta \sin \theta$ and $q(\theta)=A \cos ^{2} \theta+B \cos \theta \sin \theta+C \sin ^{2} \theta$.

The origin is a center for (1) if and only if every solution in a neighborhood of $r=0$ is a $2 \pi$-periodic solution for the differential equation

$$
\frac{d r}{d \theta}=p(\theta) r^{2}+q(\theta) r^{3} .
$$

Let $r(\theta, c)$ be the solution of $(2)$ with $r(0, c)=c$. For small $c$, we write

$$
r(\theta, c)=\sum_{n=1}^{\infty} a_{n}(\theta) c^{n},
$$

where $a_{1}(0)=1$ and $a_{n}(0)=0$ for $n>1$. The origin is a center if and only if $a_{1}(2 \pi)=1$, and $a_{n}(2 \pi)=0$ for all $n>1$. Substituting (3) into (2) and equating the coefficients of $c$ yield

$$
\dot{a}_{n}=p \sum_{i+j=n} a_{i} a_{j}+q \sum_{i+j+k=n} a_{i} a_{j} a_{k} .
$$

Received by the editors April 1, 1997.

1991 Mathematics Subject Classification. Primary 34C25; Secondary 34C05, 34C23.

(C)1998 American Mathematical Society 
Solving these equations recursively gives

$$
a_{1}=1, a_{2}=\bar{p}, a_{3}=\bar{p}^{2}+\bar{q}, a_{4}=\bar{p}^{3}+2 \bar{p} \bar{q}+\overline{\bar{p}} q
$$

and

$$
a_{5}=\bar{p}^{4}+3 \bar{p}^{2} \bar{q}+\bar{p}^{2} q+2 \bar{p} \overline{\bar{p}} q+\frac{3}{2} \bar{q}^{2}
$$

where a bar over a function denotes its indefinite integral. The functions $a_{2}$ and $a_{4}$ are integrals of polynomials of odd degrees; hence, they are $2 \pi$-periodic. Therefore, the first two necessary conditions for a center are $a_{3}(2 \pi)=0$ and $a_{5}(2 \pi)=0$. We have

If $A+C=0$, then

$$
a_{3}(2 \pi)=\pi(A+C) .
$$

$$
a_{5}=-\frac{\pi}{2}\left(A \alpha^{2}+B \alpha \beta-A \beta^{2}\right) .
$$

Now, we prove that these conditions are also sufficient. If $\alpha \beta \neq 0$, then straightforward manipulations imply that $q=\frac{-A}{\alpha \beta} p \bar{p}$. It follows inductively from (4) that $\dot{a}_{n}=p F(\bar{p})$, for some continuous function $F$. Therefore, $a_{n}(2 \pi)=\bar{F}(\bar{p}(2 \pi))-$ $\bar{F}(\bar{p}(0))=0$. If $\alpha=A=0$ and $\beta \neq 0$, then $q=-\frac{B}{\beta} p \bar{p}$; when $\beta=A=0$ and $\alpha \neq 0$, then $q=-\frac{B}{\alpha} p \bar{p}$. In the case $\alpha=\beta=0$, the equation has the form $\frac{d r}{d \theta}=q(\theta) r^{3}$. Since $q$ has mean value zero, the solutions of this equation are $2 \pi$-periodic.

The idea of this proof is the same one used in [1] for systems with homogeneous nonlinearities.

\section{REFERENCES}

[1] M.A.M. Alwash and N.G. Lloyd, Non-autonomous equations related to polynomial twodimensional systems, Proc. Royal Soc. Edinburgh, 105A(1987), 129-152. MR 88i:58145

[2] C.B. Collins, Conditions for a center in a simple class of cubic system, Differential and Integral Equations, 10(2)(1997), 333-356. CMP 97:05

Department of Mathematics, University of California, Los Angeles, California 90095

E-mail address: alwash@math.ucla.edu 